\title{
Correction to: Software-Defined Networking: Open-Source Alternatives for Small to Medium Sized Enterprises
}

Paul Thornley and Maryam Bagheri

Correction to:

Chapter "Software-Defined Networking: Open-Source

Alternatives for Small to Medium Sized"

in: L. Barolli et al. (Eds.): Advanced Information

Networking and Applications, LNNS 227,

https://doi.org/10.1007/978-3-030-75078-7_20

In the original version of the Chapter, the following correction have been incorporated: The chapter title "Software-Defined Networking: Open-Source Alternatives for Small to Medium Sized" has been changed to "Software-Defined Networking: Open-Source Alternatives for Small to Medium Sized Enterprises". 